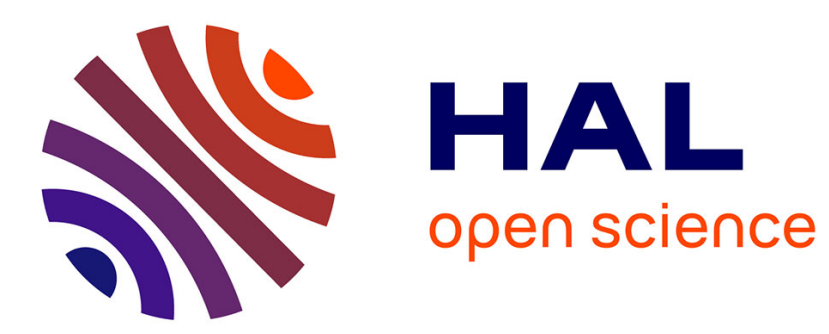

\title{
A quadratic complexity eigenspace technique for blind SIMO channel identification
}

\author{
Houcem Gazzah, Jean-Pierre Delmas
}

\section{To cite this version:}

Houcem Gazzah, Jean-Pierre Delmas. A quadratic complexity eigenspace technique for blind SIMO channel identification. ISSSE 2012: International Symposium on Signals, Systems and Electronics, Oct 2012, Potsdam, Germany. pp.1 - 5, 10.1109/ISSSE.2012.6374337 . hal-01299280

\section{HAL Id: hal-01299280 \\ https://hal.science/hal-01299280}

Submitted on 16 Dec 2020

HAL is a multi-disciplinary open access archive for the deposit and dissemination of scientific research documents, whether they are published or not. The documents may come from teaching and research institutions in France or abroad, or from public or private research centers.
L'archive ouverte pluridisciplinaire HAL, est destinée au dépôt et à la diffusion de documents scientifiques de niveau recherche, publiés ou non, émanant des établissements d'enseignement et de recherche français ou étrangers, des laboratoires publics ou privés. 


\section{A QUADRATIC COMPLEXITY EIGENSPACE TECHNIQUE FOR BLIND SIMO CHANNEL IDENTIFICATION}

\author{
Houcem Gazzah \\ Dept. of Elec. and Computer Engineering \\ University of Sharjah, 27272, UAE \\ hgazzah@sharjah.ac.ae
}

\author{
Jean-Pierre Delmas \\ Telecom SudParis, UMR CNRS 5157 \\ 91011 Evry, France \\ jean-pierre.delmas@it-sudparis.eu
}

\begin{abstract}
Eigenspace techniques are very popular techniques for blind channel identification, but are ones with a large complexity, cubic in the channel order. The newly introduced channel compaction is a signal processing technique that consists in using small-sized linear transformations to progressively force to zero some of the channel coefficients. As such, channel compaction was used to develop the first (and, up to now, the only) blind channel equalization technique with a quadratic complexity. In this paper, we apply blind compaction to develop a new blind identification technique, the first to have a quadratic complexity. Simulation tests show that the low-complexity compaction-based blind identification performs quite similarly to the most referenced existing eigenspace blind identification techniques.
\end{abstract}

\section{INTRODUCTION}

When input is known, estimating the impulse response of an $M$-order linear channel is often conducted using the well known Levinson algorithm [1, Chap. 11] whose complexity is quadratic, i.e. in $O\left(M^{2}\right)$, thanks to the Toeplitz-structure of the data matrix. When input is not known, the so-called blind identification relies only on the channel outputs. This is an interesting situation in communication systems because more bandwidth is made available to the user. Algorithms to achieve blind identification are based on high-order statistics (HOS), or, more preferably, on second-order statistics (SOS) that are much easier to estimate. They are used by the socalled eigenspace (or subspace) techniques to built quadratic cost functions whose minimization does not suffer from local convergence problems, as happens with HOS-based techniques [2]. On the negative side, eigenspace techniques assume the receiver to use many antennas and/or sample data at a rate higher than the baud-rate [3]. More seriously, complexity is in $O\left(M^{3}\right)$, as a direct consequence of inversion, pseudo-inversion, or (eigen/singular) vector decomposition (EVD/SVD) typically performed by SOS techniques.

It is only recently that a blind eigenspace channel processing technique was proposed that has a quadratic complexity.
It is based on the idea, called channel compaction, that consists in finding an invertible linear transformation to force to zero some leading and/or trailing coefficients of the channel impulse response. These transformations are derived from the correlation coefficients with large time lags. Such correlation coefficients are rank-deficient matrices and depend on some of the leading and/or trailing coefficients. A vector orthogonal to such a matrix is also orthogonal to these particular channel taps. This elementary step serves as the starting point to a low-complexity blind zero-forcing equalization technique that progressively suppresses inter-symbol interference [4].

Reduction in complexity is very desired because $M$ is large for band-limited channels [5]. We propose a quadratic complexity channel identification algorithm using the compaction principle. Since identification requires twice as much iteration as equalization, error propagation is severe to the point that estimation performance in realistic conditions may be unpractical. An other reason is that we handle sub-blocks of the correlation matrix that depend on the leading and trailing channel coefficients, which are typically small for bandlimited channels [5]. As such, these sub-blocks are difficult to estimate in the presence of noise. As a solution, we propose a channel output pre-processing step, with the objective of rendering channel coefficients of comparable norm. This preprocessing has low complexity, but shows dramatic impact on identification performance, made, by this means, comparable to what prevails for existing eigenspace techniques.

The paper is organized as follows. The multi-channel setting and the associated observation model is detailed in Sec. 2. The identification algorithm is described in Sec. 3 and some implementation issues are discussed in Sec. 4. Simulation results are displayed and discussed in Sec. 5 and a conclusion is given in Sec. 6 .

\section{THE DATA MODEL}

We adopt the well-known Single-Input Multiple-Output (SIMO) channel where the transmitted data $s(t)$ is collected by a set of $C$ antennas. It delivers a $C$-dim output $\mathbf{y}(t) \stackrel{\text { def }}{=}\left[y^{(1)}(t), \cdots, y^{(C)}(t)\right]^{T}$, where $y^{(c)}(t)$ is the snapshot 
collected by the $c$-th antenna at time index $t$. The propagation from the transmitter to the $c$-th receiving antenna is modeled as an impulse response $\mathbf{h}^{(c)}=\left[h_{0}^{(c)}, \cdots, h_{M}^{(c)}\right]^{T}$ where $M$ is the largest order among all impulses responses $\mathbf{h}^{(c)}$, $c=1, \cdots, C$. The observation model is

$$
\begin{aligned}
& \mathbf{y}(t)=\mathbf{H}[s(t), \cdots, s(t-M)]^{T}+\mathbf{n}(t) \\
& \stackrel{\text { def }}{=} \\
& \mathbf{x}(t)+\mathbf{n}(t) .
\end{aligned}
$$

In $\mathbf{H} \stackrel{\text { def }}{=}\left[\mathbf{h}_{0}, \cdots, \mathbf{h}_{M}\right]$, the $(k+1)$-th column $\mathbf{h}_{k} \stackrel{\text { def }}{=}\left[h_{k}^{(1)}, \cdots, h_{k}^{(C)}\right]^{T}$ is the $k$-th tap of the SIMO channel. The noise vector $\mathbf{n}(t)$ is defined analogously to the output vector $\mathbf{y}(t)$. We stack $L$ successive outputs into the $C L$-dim vector

$$
\begin{aligned}
& \mathbf{y}_{L}(t) \stackrel{\text { def }}{=}\left[\mathbf{y}^{T}(t) \cdots \mathbf{y}^{T}(t-L+1)\right]^{T} \\
= & \mathbf{T}_{L}\{\mathbf{H}\}[s(t), \cdots, s(t-M-L+1)]^{T}+\mathbf{n}_{L}(t),
\end{aligned}
$$

where $\mathbf{T}_{L}\{\mathbf{H}\} \stackrel{\text { def }}{=}\left[\begin{array}{cccc}\mathbf{H} & \mathbf{0} & \cdots & \mathbf{0} \\ \mathbf{0} & \mathbf{H} & \cdots & \mathbf{0} \\ \ddots & & \\ \mathbf{0} & \cdots & \mathbf{0} & \mathbf{H}\end{array}\right]$ is the $C L \times(M+L)$ channel filtering matrix, $\mathbf{0}$ is $C$-dim zero vector, and $\mathbf{n}_{L}(t)$ is defined similarly as $\mathbf{y}_{L}(t)$. We define $\sigma_{s}^{2}$ as the power of the source symbols and $\sigma_{n}^{2}$ as the power of the noise snapshots. Then, the channel correlation matrices $\mathbf{R}_{L}^{y} \stackrel{\text { def }}{=}$ $\mathbf{E}\left[\mathbf{y}_{L}(t) \mathbf{y}_{L}^{H}(t)\right]$ and $\mathbf{R}_{L} \stackrel{\text { def }}{=} \mathbf{E}\left[\mathbf{x}_{L}(t) \mathbf{x}_{L}^{H}(t)\right]$ verify

$$
\begin{aligned}
& \mathbf{R}_{L}^{y}=\mathbf{R}_{L}+\sigma_{n}^{2} \mathbf{I}, \\
& \mathbf{R}_{L}=\sigma_{s}^{2} \mathbf{T}_{L}\{\mathbf{H}\} \mathbf{T}_{L}\{\mathbf{H}\}^{H} .
\end{aligned}
$$

The above holds assuming (as commonly the case in SOSbased techniques $[3,6,7]$ ) that we have i.i.d., zero-mean source symbols uncorrelated from the white noise. The block Toeplitz-structured correlation matrix $\mathbf{R}_{L}$ is made of correlation coefficients $\boldsymbol{\Gamma}_{k} \stackrel{\text { def }}{=} \mathbf{E}\left[\mathbf{x}(t+k) \mathbf{x}^{H}(t)\right]$, possibly not zero for $k=0, \cdots, M$. They are identical to the noise-corrupted correlation coefficients $\boldsymbol{\Gamma}_{k}^{y} \stackrel{\text { def }}{=} \mathbf{E}\left[\mathbf{y}(t+k) \mathbf{y}^{H}(t)\right]$ except for $\boldsymbol{\Gamma}_{0}^{y}=\boldsymbol{\Gamma}_{0}+\sigma_{n}^{2} \mathbf{I}$

\section{THEORETICAL DEVELOPMENT}

The easy-to-obtain relationship

$$
\boldsymbol{\Gamma}_{k}=\left[\mathbf{h}_{k}, \cdots, \mathbf{h}_{M}\right]\left[\mathbf{h}_{0}, \cdots, \mathbf{h}_{M-k}\right]^{H}
$$

is useful to highlight some properties of the left and right kernels of the opportunistically small-sized $\boldsymbol{\Gamma}_{k}$. We can assume, with probability one, that any $C$ columns of $\mathbf{H}$ are linearly independent so that the rank of $\boldsymbol{\Gamma}_{k}$ is equal to $\min \{M-k+$ $1, C\}$. We assume, without any loss of generality, $C$ to be an even number. Blind channel compaction is based on the kernel properties of the $\boldsymbol{\Gamma}_{k}$ that are rank-deficient, and in particular the one with rank $C / 2$. Its left and right orthogonal vectors will be used to built up an invertible transformation matrix with interesting properties.

If $M+1 \geq C / 2$, rank of $\boldsymbol{\Gamma}_{K}$ is $C / 2$ for $K \stackrel{\text { def }}{=}$ $M+1-C / 2$. SVD of $\Gamma_{K}$ allows one to obtain $C / 2$ vectors left (resp. right) orthogonal to $\left[\mathbf{h}_{K}, \cdots, \mathbf{h}_{M}\right]$ (resp. to $\left.\left[\mathbf{h}_{0}, \cdots, \mathbf{h}_{C / 2-1}\right]\right)$, that are stacked as columns of the matrix $\mathbf{U}^{(1)}\left(\right.$ resp. $\left.\mathbf{V}^{(1)}\right)$. We assume

$$
M+1 \geq C
$$

so that $C / 2-1<K$. Consequently, $\left[\mathbf{h}_{K}, \cdots, \mathbf{h}_{M}\right]$ and $\left[\mathbf{h}_{0}, \cdots, \mathbf{h}_{C / 2-1}\right]$ do not have any column in common. As a result, kernels $\mathbf{U}^{(1)}$ and $\mathbf{V}^{(1)}$ are not related to each other. Together, they form an invertible matrix $\left[\mathbf{U}^{(1)}, \mathbf{V}^{(1)}\right]^{H}$ by means of which the SIMO channel is converted into a zeropadded one

$$
\left[\mathbf{U}^{(1)}, \mathbf{V}^{(1)}\right]^{H} \mathbf{H} \stackrel{\text { def }}{=}\left[\begin{array}{c}
\mathbf{H}^{\text {left }}, \mathbf{0}_{C / 2, C / 2} \\
\mathbf{0}_{C / 2, C / 2}, \mathbf{H}^{\text {right }}
\end{array}\right]
$$

where $\mathbf{H}^{\text {left }} \stackrel{\text { def }}{=}\left(\mathbf{U}^{(1)}\right)^{H}\left[\mathbf{h}_{0} \cdots \mathbf{h}_{K-1}\right]$ and $\mathbf{H}^{\text {right }} \stackrel{\text { def }}{=}$ $\left(\mathbf{V}^{(1)}\right)^{H}\left[\mathbf{h}_{C / 2} \cdots \mathbf{h}_{M}\right]$ are $C / 2 \times K$. Individual rows in (1) have order $K-1$ but, since none of the columns is strictly zero, the right hand side of (1) does not represent a channel with a smaller (than $M$ ) order. A few steps are needed before we can identify such a channel.

By using [4, Eq. (3)], we can prove that

$$
\begin{aligned}
& {\left[\mathbf{0}_{C M, C / 2}, \mathbf{I}_{C M}, \mathbf{0}_{C M, C / 2}\right]\left(\mathbf{I} \otimes\left[\mathbf{U}^{(1)}, \mathbf{V}^{(1)}\right]^{H}\right)} \\
& \mathbf{R}_{M+1}\left(\mathbf{I} \otimes\left[\mathbf{U}^{(1)}, \mathbf{V}^{(1)}\right]\right)\left[\mathbf{0}_{C M, C / 2}, \mathbf{I}_{C M}, \mathbf{0}\right]^{T} \\
& =\mathbf{T}_{M}\left\{\mathbf{H}^{(1)}\right\} \mathbf{T}_{M}^{H}\left\{\mathbf{H}^{(1)}\right\},
\end{aligned}
$$

where $\mathbf{H}^{(1)} \stackrel{\text { def }}{=}\left[\begin{array}{c}\mathbf{0}_{C / 2, C / 2-1}, \mathbf{H}^{\text {right }} \\ \mathbf{H}^{1 \mathrm{eft}}, \mathbf{0}_{C / 2, C / 2-1}\end{array}\right]$ is an $(M-1)$-order SIMO channel in the strict sense. Clearly, we can manipulate the SOS of the original $M$-order SIMO to obtain the SOS of an equivalent $(M-1)$-order SIMO channel. Furthermore, the SOS of the new channel can be obtained from those of the original one be means of the relationship [4]

$$
\begin{gathered}
{\left[\boldsymbol{\Gamma}_{0}^{(1)}, \cdots, \boldsymbol{\Gamma}_{M-1}^{(1)}\right]=\left[\left[\begin{array}{c}
\left(\mathbf{V}^{(1)}\right)^{H} \boldsymbol{\Gamma}_{0} \\
\left(\mathbf{U}^{(1)}\right)^{H} \boldsymbol{\Gamma}_{1}^{H}
\end{array}\right] \mathbf{V}^{(1)},\right.} \\
\left.\left[\begin{array}{c}
\left(\mathbf{V}^{(1)}\right)^{H} \boldsymbol{\Gamma}_{1} \\
\left(\mathbf{U}^{(1)}\right)^{H}{ }^{\boldsymbol{\Gamma}_{0}}
\end{array}\right]\left[\mathbf{U}^{(1)}, \mathbf{V}^{(1)}\right], \cdots,\left[\begin{array}{c}
\left(\mathbf{V}^{(1)}\right)^{H} \boldsymbol{\Gamma}_{M} \\
\left(\mathbf{U}^{(1)}\right)^{H} \boldsymbol{\Gamma}_{M-1}
\end{array}\right] \mathbf{U}^{(1)}\right]
\end{gathered}
$$

We proceed iteratively and obtain, each time, an equivalent SIMO channel with an order decremented by 1 . For instance, the next step is about transforming the $(M-1)$ order SIMO channel $\mathbf{H}^{(1)}$ into an equivalent $(M-2)$-order 
SIMO channel $\mathbf{H}^{(2)}$. This time, compaction does not require any SOS-derived transformation. Simple lines and columns permutation leads to a relationship similar to (1), see [4]. Progressively, we form a sequence of SIMO channels $\mathbf{H}^{(1)}, \cdots \mathbf{H}^{(M)}$ with a number of coefficients, $M, \cdots, 1$, respectively. These channels are not directly accessible but their respective $\operatorname{SOS} \boldsymbol{\Gamma}_{0}^{(i)}, \cdots, \boldsymbol{\Gamma}_{M-i}^{(i)}, i=1, \cdots, M$. The very last one corresponds to a zero order channel and its only non-zero correlation coefficient is

$$
\boldsymbol{\Gamma}_{0}^{(M)} \stackrel{\text { def }}{=} \mathbf{H}^{(M)}\left(\mathbf{H}^{(M)}\right)^{H},
$$

where $\mathbf{H}^{(M)}$ is, actually, a $C$-dim vector. EVD of $\boldsymbol{\Gamma}_{0}^{(M)}$ allows us to estimate $\mathbf{H}^{(M)}$ up to an unknown multiplicative constant. Such ambiguity is inherent to blind SIMO identification. Once $\mathbf{H}^{(M)}$ is estimated, $\mathbf{H}^{(M-1)}, \ldots, \mathbf{H}^{(1)}$ and, ultimately, $\mathbf{H}$ are reconstructed, up to the same ambiguity.

\section{DISCUSSION}

\subsection{Pre-processing}

A weak point is that the proposed algorithm starts with the SVD of $\boldsymbol{\Gamma}_{K}$, where $K$ is slightly lower than $M$. This $\boldsymbol{\Gamma}_{K}$ is function of a few leading and trailing channel coefficients. For band-limited channels, such coefficients are very likely to be weak [5] so that the estimation of $\boldsymbol{\Gamma}_{K}$ is sensitive to noise and its SVD is badly-conditioned.

Hence, prior to applying the above blind identification procedure, we propose a pre-processing step to convert the original SIMO channel, for which coefficients may have very unequal magnitude, into an equivalent SIMO channel for which they are bounded. This pre-processing step is not computation consuming, nor sensitive to noise because it is based on $\boldsymbol{\Gamma}_{0}$ which, in contrast, is full column rank and highly likely to be well-conditioned.

Having assumed $C \leq M+1$, the SVD of $\mathbf{H}$ is given by $\mathbf{H}=\mathbf{U}[\mathbf{D}, \mathbf{0}] \mathbf{V}^{H}$, where $\mathbf{U}$ and $\mathbf{V}$ are unitary matrices and $\mathbf{D}$ is diagonal. Both $\mathbf{U}$ and $\mathbf{D}$ can be blindly estimated from the EVD of $\boldsymbol{\Gamma}_{0}=\mathbf{H} \mathbf{H}^{H}=\mathbf{U D}^{2} \mathbf{U}^{H}$. Clearly, the following transformation $\mathbf{D}^{-1} \mathbf{U}^{H}$ leads to an equivalent SIMO channel $[\mathbf{I}, \mathbf{0}] \mathbf{V}^{H}$. The newly obtained SIMO channel has the same order $M$ but its coefficients are upper-bounded. As a consequence of $\mathbf{V}$ being unitary, the magnitude of each column of $[\mathbf{I}, \mathbf{0}] \mathbf{V}^{H}$ is smaller than $\sqrt{C /(M+1)}$. This pre-processing will show a dramatic impact on the performance of the proposed algorithm.

\subsection{Algorithm Description}

Consider now sample estimates of $\mathbf{E}\left[\mathbf{y}(n) \mathbf{y}^{H}(n-k)\right], k=$ $0, \cdots, M$ obtained from a finite number of snapshots $\mathbf{y}(t)$. These estimates are still denoted by $\boldsymbol{\Gamma}_{k}$. The identification algorithm is as follows:
1. Removing the noise contribution: Noise power $\sigma_{n}^{2}$ is estimated using the low-complexity procedure described in $\left[4\right.$, Sec. V-B], by $\hat{\sigma_{n}^{2}}$ defined as the average of the $C-1$ lowest eigenvalues of $\boldsymbol{\Gamma}_{0}+\sum_{k=1}^{M}\left[\boldsymbol{\Gamma}_{k}+\boldsymbol{\Gamma}_{k}^{H}\right]$. Update $\boldsymbol{\Gamma}_{0}$ as $\boldsymbol{\Gamma}_{0}-\hat{\sigma_{n}^{2}} \mathbf{I}$.

2. Pre-processing: Perform EVD of the $\boldsymbol{\Gamma}_{0}$ to obtain eigenvectors $\mathbf{q}_{1}, \cdots, \mathbf{q}_{C}$ and their respective eigenvalues $q_{1}, \ldots, q_{C}$. Initialize:

$\mathbf{W}^{(0)}=\operatorname{Diag}\left(1 / \sqrt{q_{1}}, \ldots, 1 / \sqrt{q_{C}}\right)\left[\mathbf{q}_{1}, \cdots, \mathbf{q}_{C}\right]^{H}$, $\boldsymbol{\Gamma}_{0}^{(0)}=\mathbf{I}$,

$\boldsymbol{\Gamma}_{k}^{(0)}=\mathbf{W}^{(0)} \boldsymbol{\Gamma}_{k}\left(\mathbf{W}^{(0)}\right)^{H}, k=1, \cdots, M$, and $i=0$.

3. Forward mode While $i<M$, repeat:

(a) Set $m=M-i$ and $k=\max \{m+1-C / 2,1+\lfloor m / 2\rfloor\}$.

(b) Perform SVD of $\boldsymbol{\Gamma}_{k}^{(i)}$. Let $\mathbf{u}_{j}$ (resp. $\mathbf{v}_{j}$ ) be the left (resp. right) unit-norm singular vector associated with the $j$-th lowest singular value. Built the $C$ square matrix $\mathbf{W}^{(i+1)}=\left[\mathbf{u}_{1}, \cdots, \mathbf{u}_{C / 2}, \mathbf{v}_{1}, \cdots, \mathbf{v}_{C / 2}\right]^{H}$.

(c) Calculate the inverse $\left[\mathbf{W}^{(i+1)}\right]^{-1}$ of $\mathbf{W}^{(i+1)}$.

(d) Calculate $\boldsymbol{\Gamma}_{0}^{(i+1)}, \cdots, \boldsymbol{\Gamma}_{m-1}^{(i+1)}$ as follows:

$$
\begin{aligned}
\boldsymbol{\Gamma}_{j}^{(i+1)}= & {\left[\left[\begin{array}{c}
\left(\mathbf{V}^{(i+1)}\right)^{H} \boldsymbol{\Gamma}_{j}^{(i)} \\
\left(\mathbf{U}^{(i+1)}\right)^{H} \boldsymbol{\Gamma}_{j-1}^{(i)}
\end{array}\right] \mathbf{V}^{(i)}\right.} \\
& {\left.\left[\begin{array}{c}
\left(\mathbf{V}^{(i+1)}\right)^{H} \boldsymbol{\Gamma}_{j+1}^{(i)} \\
\left(\mathbf{U}^{(i+1)}\right)^{H} \boldsymbol{\Gamma}_{j}^{(i)}
\end{array}\right] \mathbf{U}^{(i)}\right], }
\end{aligned}
$$

for $j=0, \cdots, m-1$ and increment $i$ by 1 .

(e) Repeat $m-k$ times:

i. Set $\mathbf{W}^{(i)}=\left[\begin{array}{cc}\mathbf{0} & \mathbf{I}_{C / 2} \\ \mathbf{I}_{C / 2} & \mathbf{0}\end{array}\right]$.

ii. Same as step $3 \mathrm{~d}$.

4. Backward mode

(a) Initialize $\hat{\mathbf{H}}^{(M)}$ as the eigenvector associated with the $i$-th largest eigenvalue of $\boldsymbol{\Gamma}_{0}^{(M)}$.

(b) For $i=M-1, \cdots, 0$, calculate $\hat{\mathbf{H}}^{(i)}=\left[\mathbf{W}^{(i+1)}\right]^{-1}\left[\begin{array}{c}\mathbf{H}_{\text {down }}, \mathbf{0}_{C / 2, C_{1}} \\ \mathbf{0}_{C / 2, C_{1}}, \mathbf{H}_{\text {up }}\end{array}\right]$ where $\mathbf{H}_{\text {up }}$ and $\mathbf{H}_{\text {down }}$ are made of, respectively, the first and last $C / 2$ rows of $\mathbf{H}^{(i+1)}$.

(c) Calculate $\left[\mathbf{W}^{(0)}\right]^{-1} \hat{\mathbf{H}}^{(0)}$ as the estimate of $\mathbf{H}$, up to an unknown multiplicative constant. 


\subsection{Identifiability}

Channel identifiability is guaranteed at the condition that all transformation matrices $\mathbf{W}^{(i)}$ are full rank. As explained earlier, the condition $K \geq(M+1) / 2$ is necessary and sufficient (with probability 1 ). In comparison, existing SOS-based techniques require a full column-rank channel filtering matrix, i.e. a minimum-phase SIMO impulse response: The (ztransforms of the) sub-channels impulse responses should not have common zeros. In practice, this can be reasonably assumed to be true (with probability 1) [3].

Nevertheless, we are tempted to study the behavior of the proposed technique applied to non-minimum-phase SIMO channels. Because each impulse response of a sub-channel of $\mathbf{H}^{(i)}$ is a linear combination of those of $\mathbf{H}^{(i-1)}$, every common zero to the latter will be a common zero of the former. As the length of the compacted channel decreases, at some stage of the algorithm, for the newly computed $\mathbf{H}^{(i)}$, all (ztransforms of all) sub-channels impulse responses are made of common zeros only, i.e. rows of $\mathbf{H}^{(i)}$ are identical up to some multiplicative constants. As a consequence, columns of $\mathbf{H}^{(i)}$ are co-linear so that rank-deficient $\boldsymbol{\Gamma}_{k}^{(i)}$ are Hermitian and, so, have identical left and right kernels, which then, violates the identifiability condition.

As a conclusion, similarly as for existing SOS-based techniques, the proposed one fails to identify a minimum-phase channel. However, the algorithm becomes instable only at the very last iterations when the compacted SIMO channel is, actually, a SISO channel corresponding to the part common to all sub-channels. This is a clear advantage of the proposed algorithm. In particular, this means that the proposed algorithm can be claimed to be the only eigenspace technique of channel shortening (examples are $[8,9]$ ) capable of handling non-minimum-phase channels.

\subsection{Computation Complexity}

First, notice that calculations involving matrices $\mathbf{W}^{(i)}$ obtained in (3(e)i) do not contribute to complexity since they merely imply row/column permutations. Consequently, only steps (3b), (3c), (3d) and (4b) [only when the involved $\mathbf{W}^{(i)}$ was computed in (3b) and not in (3(e)i)] are taken into consideration for the operations count. For practical reasons, it is plausible to assume that the number $C$ of sensors is limited and that only the channel order $M$ is eventually large. As a consequence, steps in the algorithm that involve SVDs, EVDs and inversions of $C$-square matrices are not what contribute the most to the computation burden because each is executed a maximum of $M$ times.

If $M$ is large enough (compared to $C$ ), $k$ in step (3a) is equal to $m+1-C / 2$ except for the last values taken by the decreasing $m$. Consequently, successive values taken by $m$ at step (3) are $M+1-C / 2, M+1-C, \cdots$ so that the loop (3) is executed $2 M / C$ times approximately, for large
$M$. The computation load is mainly due to matrix multiplications in steps (3d) and (4b). We estimate it, bearing in mind that the product of $m_{1} \times m_{2}$ matrix by an $m_{2} \times m_{3}$ matrix results in $O\left(2 m_{1} m_{2} m_{3}\right)$ flops [10]. On one hand, step (3d) involves $O\left(6 C^{3}(m+1)\right)$ flops, where $m=M-i C / 2$ and $i=0, \cdots, 2 M / C$, hence, leading to a load of approximately $O\left(6 C^{2} M^{2}\right)$. On the other hand, step (4b) involves $O\left(2 C^{2}(M-i)\right)$ flops for $i+1=1,1+C / 2,1+C, \cdots, M$ leading to a computation load of of $O\left(2 C M^{2}\right)$. The total computation load is, hence, given by $O\left(2(1+3 C) C M^{2}\right)$, which is quadratic in $M$, as claimed.

\section{SIMULATIONS}

A series of simulations has been conducted to compare the proposed algorithm to the main eigenspace blind identification techniques. We use the SIMO channel from [6] with $C=4$ sub-channels and an order $M=4$. It is driven by unit-variance i.i.d. 4-QAM symbols and corrupted by additive white Gaussian noise. The channel observation SNR is defined as $\mathbf{E}\left[\|\mathbf{x}(t)\|^{2}\right] / \mathbf{E}\left[\|\mathbf{n}(t)\|^{2}\right]=$ $\sigma_{s}^{2} \sum_{m=0}^{M}\left\|\mathbf{h}_{m}\right\|^{2} /\left(C \sigma_{n}^{2}\right)$.

A channel estimate $\hat{\mathbf{h}}$ approximates the exact impulse response $\mathbf{h} \stackrel{\text { def }}{=}\left[\mathbf{h}_{0}^{T}, \cdots, \mathbf{h}_{M}^{T}\right]^{T}$, up to a scaling and rotation ambiguity. The estimation accuracy is evaluated in terms of the identification Mean Square Error (MSE) defined [11] as

$$
1-\left(\frac{\left|\mathbf{h}^{H} \hat{\mathbf{h}}\right|}{\|\mathbf{h}\|\|\hat{\mathbf{h}}\|}\right)^{2}
$$

We tested the proposed technique in comparison with some among the most referenced eigenspace blind identification algorithms, namely, the Sub-Space (SS) technique [6], the Outer Product Decomposition (OPD) [7] technique and the Linear Prediction (LP) technique [12].

Identification results, averaged over 100 Monte Carlo runs, are reported in Fig. 1 as function of an increasing number of snapshots. They show that the proposed technique is capable of estimating the channel response with an accuracy comparable to that of the existing algorithms. The pre-processing step described in Sec. 4.1 is very effective in improving the performance of the proposed algorithm. The gap to the existing algorithms is reduced when the number of snapshots increases. Results reported in [4] show compaction-based equalization to perform very closely to existing algorithms. This contrasts with blind channel identification, where channel compaction is clearly less efficient. In fact, compaction-based blind equalization is a forwardonly procedure, while compaction-based blind identification is a forward-backward procedure, resulting in twice as many steps and, hence, more error propagation occurs. 


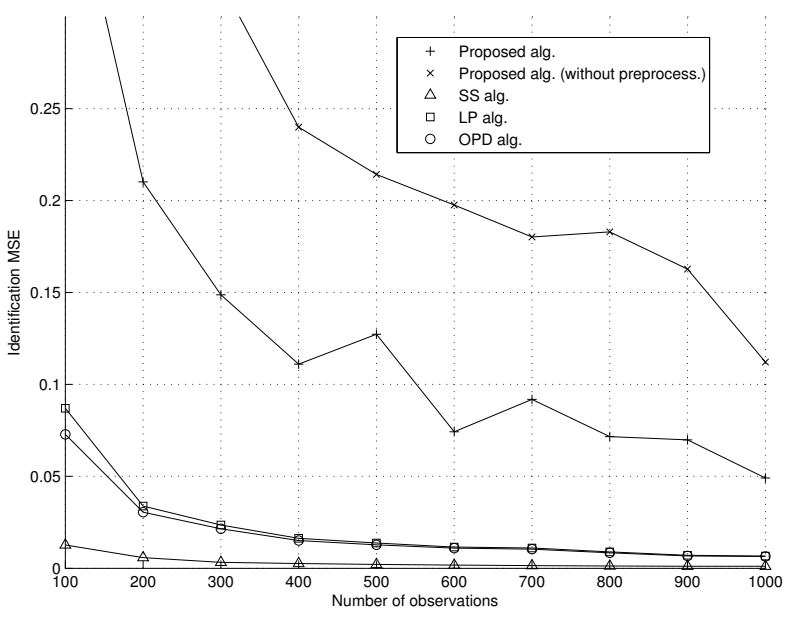

(a) $\mathrm{SNR}=20 \mathrm{~dB}$

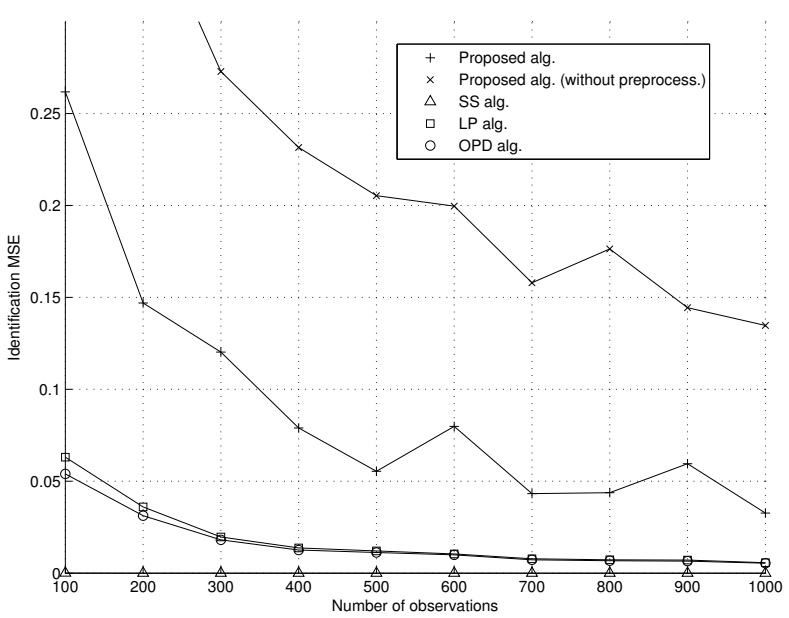

(b) $\mathrm{SNR}=40 \mathrm{~dB}$

Fig. 1. Identification MSE as function of the number of snapshots.

\section{CONCLUSION}

Using the recently-introduced principle of channel compaction [4], we develop an blind channel identification algorithm with an unprecedented quadratic complexity, in $O\left(M^{2}\right)$, where $M$ is the channel order. In the first step (forward mode), channel order is reduced progressively from $M$ to zero, and channel SOS are updated in the process. The response of the zero-order channel is trivially estimated from the channel SOS. Then, a second step (backward mode) is started where the channel taps are progressively reconstructed until reaching (an estimate of) the original (physical) channel. Simulation tests are conducted to prove the efficiency of the proposed method under realistic observation conditions.

\section{REFERENCES}

[1] B. Farhang-Boroujeny, Adaptive Filters: Theory and Applications, John Wiley \& Sons, 1998.

[2] D. N. Godard, "Self-recovering equalization and carrier tracking in two-dimentional data communication systems," IEEE Transactions on Communications, vol. COM-28, no. 11, pp. 1867-1875, Nov. 1980.

[3] L. Tong, G. Xu and T. Kailath, "Blind identification and equalization based on second-order statistics : a time domain approach," IEEE Trans. Inform. Theory, vol. 40, pp. 340-349, Mar. 1994.

[4] H. Gazzah, "SOS-based blind channel equalization with quadratic complexity," IEEE Trans. Signal Processing, vol. 59, no. 2, pp. 837-841, Feb. 2011.

[5] J.-P. Delmas, H. Gazzah, A. P. Liavas and P. A. Regalia, "Statistical analysis of some second-order methods for blind channel identification/equalization with respect to channel undermodeling," IEEE Trans. Signal Processing, vol. 48, no. 7, pp. 1984-1998, July 2000.

[6] E. Moulines, P. Duhamel, J.-F. Cardoso and S. Mayrargue, "Subspace methods for the blind identification of multichannel FIR filters," IEEE Trans. Signal Processing, vol. 43, pp. 516-525, Feb. 1995.

[7] Zhi Ding, "Matrix outer product decomposition method for blind multiple channel identification," IEEE Transactions on Signal Processing, vol. 45, no. 12, pp. 30533061, Dec. 1997.

[8] T. Miyajima and Z. Ding, "Second-order statistical approaches to channel shortening in multicarrier systems," IEEE Trans. Signal Processing, vol. 52, pp. 3253-3264, Nov. 2004.

[9] H. Gazzah and Steve McLaughlin, "An SOS-based blind channel shortening algorithm," in Proc. Int. Conf. Acoustics, Speech, Signal Processing (ICASSP), 2006, pp. 345348.

[10] G. H. Golub and C. F. Van Loan, Matrix Computations, North Oxford Academic, 1983.

[11] H. Gazzah, P. A. Regalia, J.-P. Delmas and K. AbedMeraim, "A blind multichannel identification algorithm robust to order overestimation," IEEE Trans. Signal Processing, vol. 50, no. 6, pp. 1449-1458, June 2002.

[12] H. Gazzah, "Optimum blind multichannel equalization using the linear prediction algorithm," IEEE Trans. Signal Processing, vol. 54, no. 8, pp. 3242-3247, Aug. 2006. 